\title{
Retroperitoneoscopic nephrectomy versus open surgery for non-tuberculous pyonephrotic nonfunctioning kidney: a single-center experience
}

\author{
Xinguang Wang, Kun Tang, Zhiqiang Chen, Hailang Liu, Ejun Peng, Ding Xia \\ Department of Urology, Tongji Hospital, Tongji Medical College, Huazhong University of Science and Technology, Wuhan, China \\ Contributions: (I) Conception and design: X Wang; (II) Administrative support: D Xia; (III) Provision of study materials or patients: H Liu, E Peng; (IV) \\ Collection and assembly of data: K Tang, H Liu, Z Chen; (V) Data analysis and interpretation: X Wang, K Tang, H Liu, Z Chen; (VI) Manuscript \\ writing: All authors; (VII) Final approval of manuscript: All authors. \\ Correspondence to: Ejun Peng; Ding Xia. No.1095, Jiefang Avenue, Qiaokou District, Wuhan 430030, China. Email: tjhmldsj@sina.com; \\ BEING1949@aliyun.com.
}

Background: Patients with obstructive pyonephrotic nonfunctioning kidney (OPNK) often require simple nephrectomy for long-term severe clinical symptoms. We aimed to analyze the outcomes of retroperitoneal laparoscopy versus open surgery for OPNK.

Methods: The study included clinical data of 69 patients with non-tuberculous OPNK from January 2015 to June 2019 in a single center. The patients were divided into laparoscopic group (LS, N=33) and open surgery group (OS, N=36). Those whose pathological findings were xanthogranulomatous inflammation or tuberculous granuloma were excluded. Statistical analysis compared the two groups in terms of basic demographic characteristics, preoperative laboratory examination results, and intraoperative and postoperative observation indicators.

Results: The results showed that non-tuberculous OPNK were more common in women (female/male =4:1). Compared with the LS group, patients in the OS group had higher white blood cells (WBC; $\mathrm{P}=0.010)$ and neutrophils $(\mathrm{P}=0.005)$ counts before surgery. The main clinical symptoms were low back pain, pyuria, and fever; among them, low back pain combined with pyuria was in the majority. More intraoperative hypotension events were observed in the OS group $(\mathrm{P}=0.007)$. Notably, subgroup analysis showed larger stone size happened in the OS group (OR 3.538, 95\% CI, 1.337, 9.208). No statistical difference was found in the duration of surgery between the two groups while the length of postoperative hospitalization and retroperitoneal drainage, and postoperative blood transfusion rate increased significantly in the OS group. Postoperative use of non-steroidal anti-inflammatory drugs was more common in the LS group, while opioid analgesics were in the OS group $(\mathrm{P}=0.0006)$. There was no statistical difference in other complications.

Conclusions: In conclusion, considering the advantages of LS in terms of postoperative blood transfusion, surgical drainage and length of hospital stay, we recommend it for non-tubercular OPNK when the stone load of pyonephrosis side was less than $280 \mathrm{~mm}^{2}$ and the preoperative WBC and neutrophil count were within the normal range.

Keywords: Non-tuberculous; pyonephrotic nonfunctioning kidney; laparoscopy; open surgery; retroperitoneoscopic nephrectomy

Submitted Nov 22, 2020. Accepted for publication Jan 29, 2021.

doi: $10.21037 /$ tau-20-1449

View this article at: http://dx.doi.org/10.21037/tau-20-1449 


\section{Introduction}

At present, the surgical treatment of upper urinary tract stones with local pyonephrosis through nephrostomy and percutaneous nephrolithotomy (PCNL) has been widely adopted internationally. However, when the cause of the disease has not been resolved for a long time and local pyonephrosis develops into a non-functional pyonephrotic kidney, surgical removal of the affected kidney is often required (1).

There was certain controversy over the better surgical approach of nephrectomy for benign lesions. Chen et al. believed both retroperitoneal and transperitoneal nephrectomy had no obvious difference in blood loss and postoperative hospital stay while other scholars found retroperitoneal nephrectomy could achieve less pain and shorter hospital stay $(2,3)$. In recent years of clinical practice, we have accumulated a series of experience in retroperitoneal laparoscopic nephrectomy. Owing to the relatively more experience, our team finally chosen the retroperitoneal approach for the current study. However, for the resection of kidneys with complicated infections, achieving the desired surgical results is always a challenge $(4,5)$. The difficulty of resection of the pyonephrotic kidney mainly comes from the blurred anatomy, severe perirenal adhesion and even the accidental injury of the large blood vessels caused by the extensive adhesion during the operation $(6,7)$. Therefore, different surgical resection methods including laparoscopic and open surgery have been tried repeatedly in different centers (1,4-10).

Until now, scholars have conducted research reports on tuberculous pyonephrotic kidney or simple non-functioning nephrectomy (1-4,9,11-13). However, as far as we know, there is few reports on the comparison of different surgical methods for calculous obstructive pyonephrotic nonfunctional nephrectomy (excluding other forms of obstruction). Significantly, this study is one of the few so far to compare the clinical outcomes of different nephrectomy methods in the treatment of non-tuberculous pyonephrotic non-functioning kidneys. Therefore, it may have certain guiding significance for improving clinical treatment. We present the following article in accordance with the STROBE reporting checklist (available at http://dx.doi.org/10.21037/tau-20-1449).

\section{Methods}

\section{Data collection}

In this study, the data of all patients who underwent simple nephrectomy from January 2015 to June 2019 were selected from the electronic medical record system of our center. A total of 69 patients underwent non-tuberculous pyonephrotic nonfunctioning nephrectomy were enrolled. The pyonephrosis was caused by urinary stones located in kidney and/or upper ureter. All included patients were divided into laparoscopic surgery group (LS group; $\mathrm{N}=33$ ) and open surgery group (OS group; $\mathrm{N}=36$ ). All participants gave their informed consent to use their data for this retrospective study involving human participants. The demographic characteristics and laboratory test results of patients before surgery were collected and registered in the Haitai electronic medical record system. The history of ipsilateral renal surgery (IRS) included PCNL, laparoscopic/ open pyelolithotomy, and flexible ureteroscopic lithotripsy. Renal function was evaluated by observing the results of renal dynamic imaging after intravenous injection of $99 \mathrm{Tcm}-$ DTP $(5,10)$. The duration of the operation started with the incision of the skin and ended with the completion of the skin suture. The diagnosis of intraoperative hypotension was based on the systolic blood pressure $<90 \mathrm{mmHg}$ for more than 10 minutes. And the evaluation of non-functioning kidney is based on any of the following points: (I) partial renal function less than $10 \%$ according to renal dynamic scan; (II) The average daily drainage volume of nephrostomy within 1 week before surgery was less than $50 \mathrm{~mL}$ and CT (Computed Tomography) examination showed that there was no residual renal function (Figures 1,2).

The study was conducted in accordance with the Declaration of Helsinki (as revised in 2013). It was approved by institutional ethnics committee of Tongji Hospital, Tongji Medical College, Huazhong University of Science and Technology (No. TJ-A20181101) and individual consent for this retrospective analysis was waived.

\section{Inclusion and exclusion criteria}

The inclusion criteria of the study were as follows: (I) the kidney was identified as a non-functioning kidney after imaging and clinical evaluation; (II) pyogenic changes or abscess formation was found in pathological sections after specimen resection. The above two conditions must be met at the same time. Exclusion criteria include: (I) pathological results support xanthogranulomatous inflammation or tuberculous granuloma; (II) those with a history of partial nephrectomy; (III) the surgical method adopted was transperitoneal, regardless of open surgery or laparoscopy; (IV) patients with kidney transplantation. 

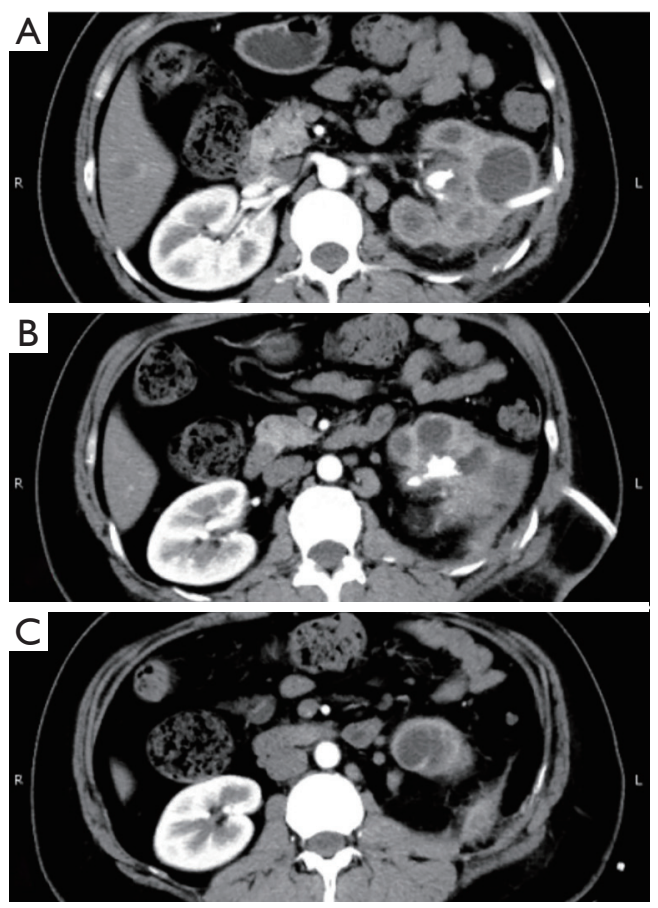

Figure 1 Enhanced CT images of the left calculous pyonephrosis after percutaneous nephrostomy. (A) At the level of the left renal hilum, left pelvic calculus with dilatation of left renal pelvis and nephric calyces can be seen, and left pararenal space catheterization; (B) in the lower pole of the left kidney, a stone of the left renal pelvis can be seen with dilation of the left renal pelvis and calyces; $(\mathrm{C})$ at the level of the lower pole of the left kidney, the left renal calyx is seen to be dilated.

\section{Retroperitoneal laparoscopic surgery technique}

Place a lateral position after general anesthesia, and raise the lumbar bridge. The first incision was selected under the posterior axillary line, and the extraperitoneal fat in the Petit triangle area (between the Gerota's fascia anteriorly and psoas fascia posteriorly) was separated with the index finger and the peritoneum was pushed to the ventral side (14). After that, the space of Petit triangle area was expanded by inflating about $800 \mathrm{~mL}$ with a self-made balloon. With the aid of the first incision, puncture with the second trocar at the tip of the 12 ribs in the anterior axillary line. Cut the skin $2 \mathrm{~cm}$ above the iliac crest and place a third trocar for camera access. When intraoperative adhesions were limited and controllable, separate the kidney outside of Gerota's fascia; otherwise, cut along the kidney capsule. Search for the ureter as an anatomical landmark for further separation, and use Hem-o-lok clips to treat the ligation and sever the renal arteries and veins. When laparoscopic surgery has no progress, it should be changed to open surgery in time. Finally, the skin incision was appropriately enlarged to complete the removal of the kidney specimen.

\section{Open surgery technique}

The open surgery was performed by professors with senior professional titles in the team. All included patients were treated through a retroperitoneal approach. Individuals who were initially assigned to open surgery and those who were transferred from laparoscopic surgery to open surgery were included in this group.

\section{Statistical analysis}

The statistical data involved in our study was analyzed by using GraphPad Prism 7.04 (GraphPad Software Corp., San Diego, California) and SPSS 25.0 (IBM Corp., New York) statistical software. The analysis of measurement data was based on $t$-test or Mann-Whitney test according to whether it satisfied normal distribution and homogeneity of variance. Categorical variables were processed by Fisher's exact test and $\mathrm{P}<0.05$ was regarded as statistically significant. Part of the data was processed by Pearson correlation analysis, and the $r$ value represented the degree of correlation. The value of $r$ was between -1 and 1 . The distribution of positive and negative values explained a positive and negative correlations. The closer the absolute value was to 1 , the higher the degree of correlation.

\section{Results}

\section{Summary of preoperative basic characteristics}

Our research showed that women accounted for an absolute high proportion of all 69 patients. There were no significant differences in demographic characteristics, previous kidney surgery history, hydronephrosis, and renal function between the two groups of LS and OS. In terms of preoperative infection-related laboratory tests, patients in the OS group had significantly increased levels of white blood cells (WBC) $(\mathrm{P}=0.010)$ and neutrophils $(\mathrm{P}=0.005)$, but there was no statistical difference in the results of urine leukocyte, nitrite and urine culture. Subgroup analysis revealed that pyonephrosis patients with renal stone larger than $280 \mathrm{~mm}^{2}$ were mostly operated on in the OS group (Table 1). The main clinical manifestations of the two groups of patients 

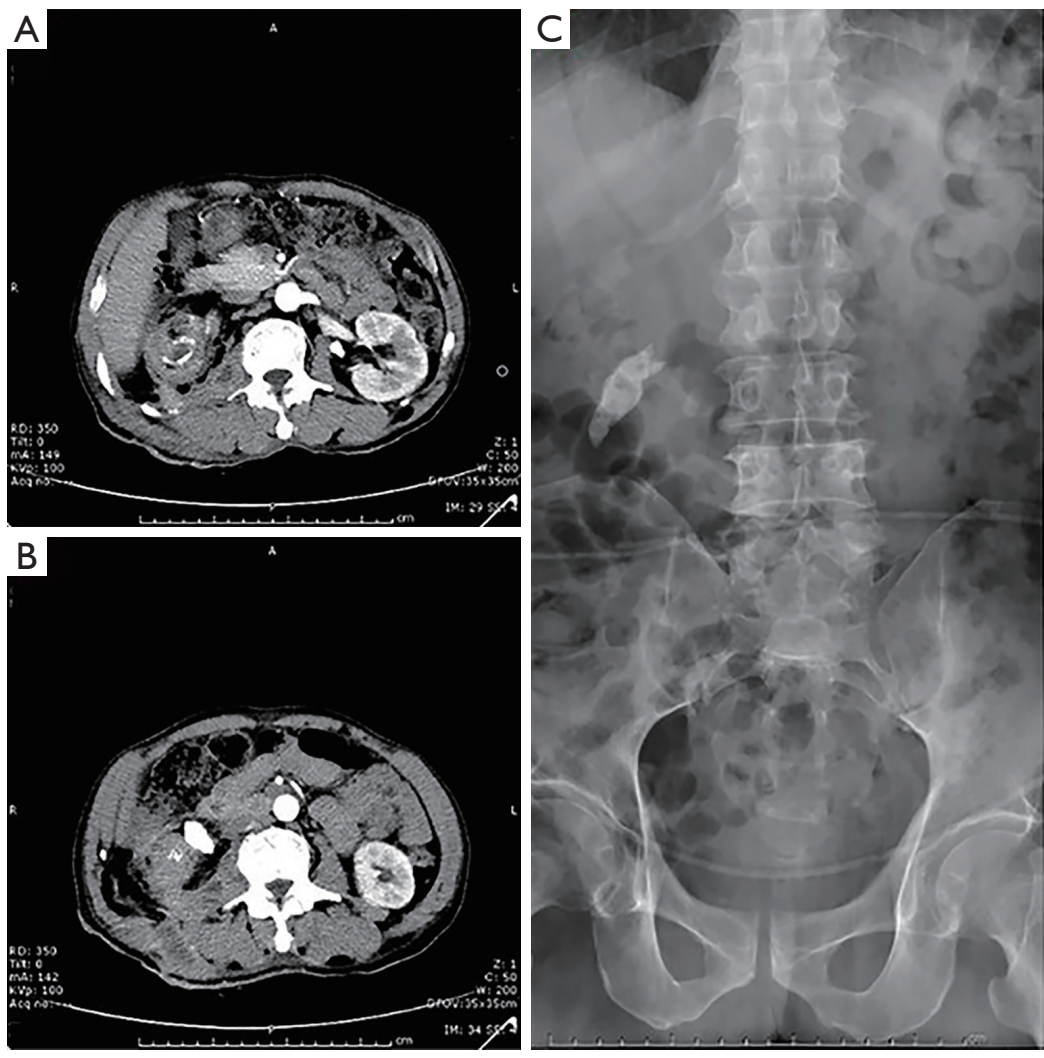

Figure 2 Enhanced CT and KUB images of the right calculous pyonephrosis before simple nephrectomy. (A) The middle pole of the right kidney, the right kidney is obviously atrophy and multiple stones accumulate in the renal collection system; unclear border around the kidney; (B) in the lower pole of the right kidney, a $23 \mathrm{~mm} \times 15 \mathrm{~mm}$ stone completely obstructs the upper ureter; (C) preoperative KUB X-ray showed highdensity nodules in the right kidney area (confirmed as stones). KUB, kidney, ureter, and bladder.

were low back pain, pyuria and fever, and low back pain combined with pyuria accounted for the highest proportion (Tables 2,3).

\section{Intraoperative and postoperative comparisons between $L S$ and OS group}

The operation duration of the two groups was up to 3.5 hours due to the relative complexity of the disease. Notably, OS group was more likely to suffer an intraoperative hypotension $(\mathrm{P}=0.007)$. There was no difference in the incidence of postoperative infectious complications (including fever greater than 38 degrees and SIRS) between the two groups. No more than two patients in each group was transferred to the intensive care unit (ICU) after surgery and recovered well. Compared with the LS, the postoperative blood transfusion rate of patients in the OS group increased significantly $(\mathrm{P}=0.0005)$. Severe postoperative complications included hyperkalemia and bleeding in 3 cases, which were effectively treated by drugs (2 patients: serum potassium 6.2 and $6.1 \mathrm{mmol} / \mathrm{L} ; 10$ units of insulin and 25 grams of glucose were infused intravenously, and sodium bicarbonate was used to correct acidosis) and surgery (1 patients: the bleeding originated from the muscular arterioles at the incision; second open operation was performed) respectively (Table 4).

In addition, there were significant differences in the application of postoperative analgesics between the two groups. The LS group tended to receive more non-steroidal anti-inflammatory drugs (NSAIDs), while the OS group was dominated by opioid analgesics $(\mathrm{P}=0.0006)$ (Figure 3). Meanwhile, the length of postoperative retroperitoneal drainage and hospital stay of patients in the OS group were significantly prolonged. Pearson correlation analysis showed that the prolonged hospital stay was mainly caused by the increased duration of retroperitoneal drainage $(r=0.914$, 95\% CI, 0.865-0.946) (Figure 4). 
Table 1 Subgroup analysis of potential relationship between perioperative indicators and different surgical methods

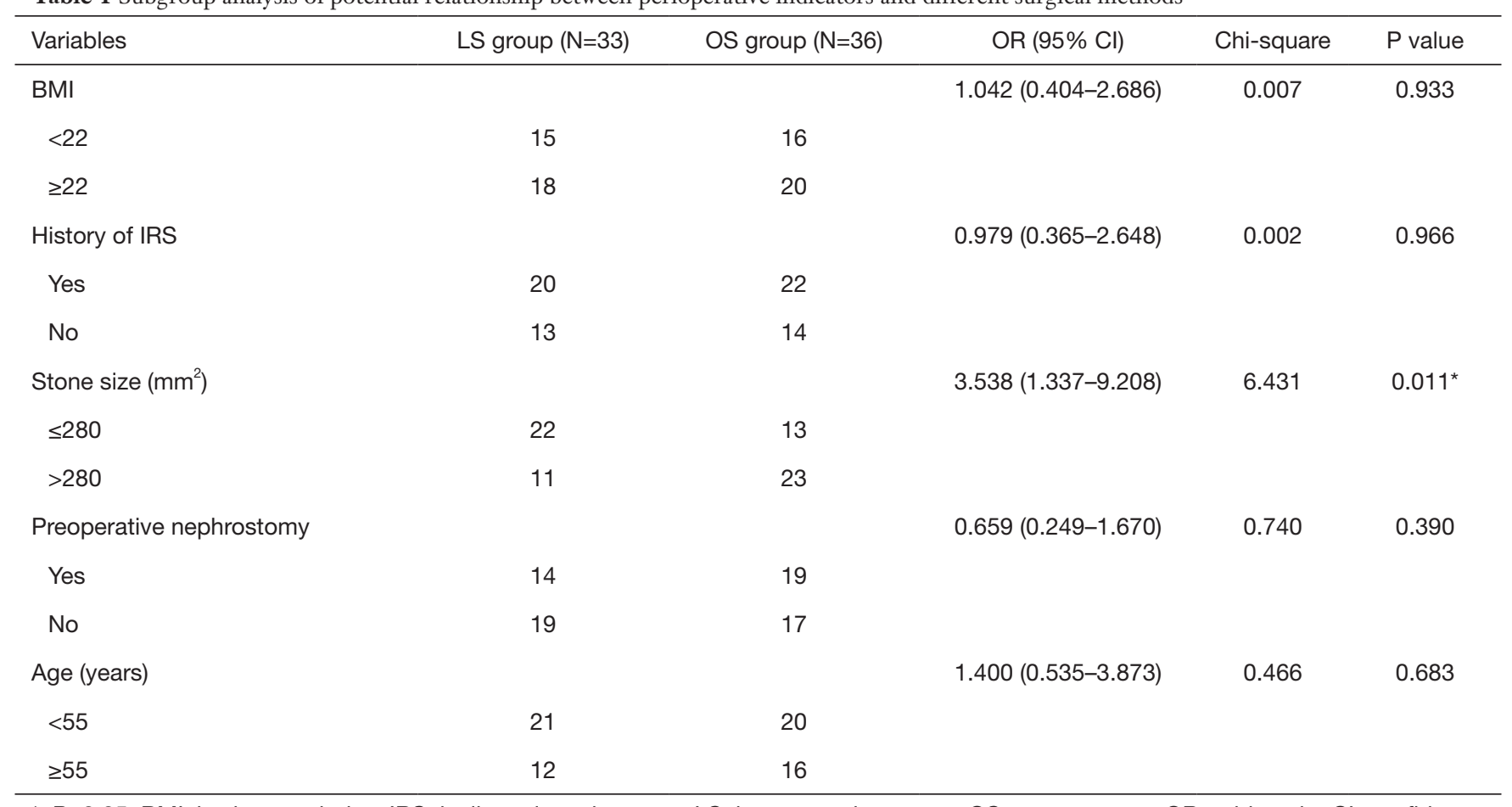

*, P<0.05. BMI, body mass index; IRS, ipsilateral renal surgery; LS, laparoscopic surgery; OS, open surgery; OR, odds ratio; Cl, confidence interval.

\section{Discussion}

Pyonephrotic kidneys were mostly caused by renal or postrenal causes, which led to extensive pyonephrotic inflammation of renal parenchyma and loss of renal function (15). Relevant articles showed that stone or obstruction at ureteropelvic junction (UPJ) accounted for a high proportion of all reasons $(10,16)$. At present, a series of studies have investigated and analyzed the surgical outcomes of simple nephrectomy for tuberculous pyonephrotic kidney $(10,16)$. This study retrospectively analyzed the nontubercular obstructive pyonephrotic nonfunctioning kidney (OPNK) over a period of 54 months in our center, and elaborated the perioperative efficacy of open surgery and retroperitoneal laparoscopic surgery.

It had previously been found that men were more likely to form stones than women, while women had a higher proportion of nonfunctioning kidneys (17). Consistent with this, our findings supported a significantly high proportion of non-tubercular OPNK in women. Both groups (OS and LS groups) had similar body mass index (BMI) in the study without significantly or extremely obese individuals. The maximum stone size of patients in both groups was greater than $15 \mathrm{~mm}$, and Mao et al. (16) confirmed that stone size greater than $10 \mathrm{~mm}$ was a risk factor for nonfunctioning kidney. The mean stone burden in the OS group was higher than that in the laparoscopic group (331.1 vs. $247.9 \mathrm{~mm}^{2}$ ) and this was supported by larger stones in OS group according to subgroup analysis. Chronic calculi, recurrent urinary tract infections, and previous surgical history all contributed to the fibrous response, resulting in numerous fibrous adhesions $(10,18)$. This undoubtedly reflected the relatively severe degree of infection and the complexity of symptoms in patients with pyonephrotic kidney disease. It presented significant challenges to intraoperative separation of perirenal tissue. The operation time in both groups reached 3.5 hours, but there was no statistical difference $(211.3 \pm 59.1$ vs. $213.1 \pm 80.6 \mathrm{~min})$. Katz et al. found that the average operative time of non-functioning kidney patients with obstruction complicated by infection caused by different reasons was $224 \mathrm{~min}(1)$, which was basically consistent with our study. Tepeler et al. believed that calculus-related nonfunctioning nephrectomy was often prolonged due to the difficulty in separating adhesions during surgery (8).

However, the relatively higher incidence of intraoperative 
Table 2 Basic characteristics of patients in the open and laparoscopic surgery group

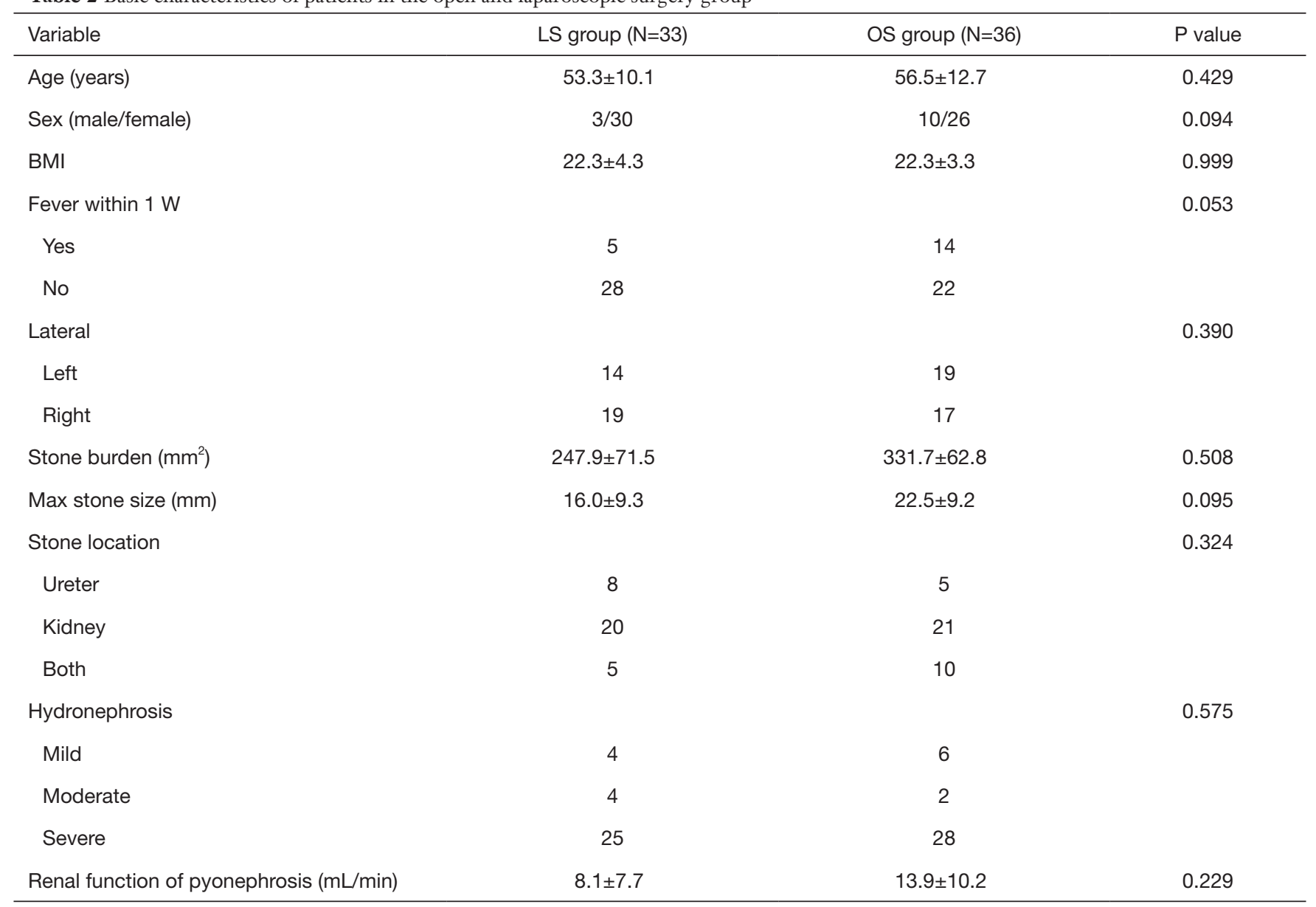

*, P<0.05. LS, laparoscopic surgery; OS, open surgery; W, week.

hypotension in the OS group (53\% \& $21 \%)$ somewhat reflected the greater degree of infection in this group. In addition, the unconventional use of intraoperative antibiotics and the broad inclusion criteria of intraoperative hypotension possibly increased the cumulative value of intraoperative hypertension events to a certain extent. Although "Simple nephrectomy" had been proposed as a standard nephrectomy for nonfunctioning nephrectomy, recently some scholars had proposed that "Simple nephrectomy is not always Simple", due to increased difficulty in dealing with inflammatory adhesion during nephrectomy $(4,5)$. This was crucial to understanding the classification of nephrectomies with complex infections such as pyonephrotic kidneys. Therefore, preoperative comprehensive and detailed assessment of surgical complexity, including surgical risks, technical difficulties and attention, was required (19).
Meanwhile, for the following reasons, the postoperative transfusion rate was significantly reduced in the retroperitoneal laparoscopic surgery group. First, the intraoperative perirenal adhesion degree of the patients in this group was relatively low, which was conducive to intraoperative resection outside of Gerota's fascia. Secondly, 8 patients $(19.5 \%)$ were transferred to open surgery due to difficulties during laparoscopic surgery, which was slightly higher than that in the study of Hemal et al. (7). Otherwise, other complications should also be avoided in the process of competing with time (20). Furthermore, laparoscopic surgery itself has the advantage of careful manipulation of deep vascular structures, to a certain extent to avoid the accidental injury of a single beam of blood vessels. Finally, after surgery, inflation of carbon dioxide was routinely terminated to observe the residual bleeding point to facilitate hemostasis (21). In addition, retroperitoneal 
Table 3 Comparison of preoperative laboratory indexes between laparoscopic and open surgery groups

\begin{tabular}{lccc}
\hline Indexes & LS group $(\mathrm{N}=33)$ & OS group $(\mathrm{N}=36)$ & $\mathrm{P}$ value \\
\hline WBC $\left(\times 10^{9} / \mathrm{L}\right)$ & $6.35 \pm 2.65$ & $9.12 \pm 3.15$ & $0.010^{\mathrm{a}}$ \\
Neutrophils $\left(\times 10^{9} / \mathrm{L}\right)$ & $4.03 \pm 2.34$ & $6.86 \pm 2.99$ & $0.005^{\mathrm{a}}$ \\
Serum creatinine $(\mu \mathrm{mol} / \mathrm{L})$ & $93.6 \pm 66.7$ & $83.4 \pm 28.2$ & 0.556 \\
CCR $\left(\mathrm{mL} / \mathrm{min} / 1.73 \mathrm{~m}^{2}\right)$ & $77.8 \pm 35.8$ & $78.8 \pm 34.5$ & 0.935 \\
Urine leukocyte $(\mathrm{n} / \mathrm{\mu L})$ & $318.4(66.1-1,212.2)$ & $271.6(17.9-5,631.6)$ & 0.731 \\
Urine culture & & & $0.406^{\mathrm{b}}$ \\
Positive & 8 & 24 & 25 \\
Negative & 25 & & \\
\hline
\end{tabular}

The interval in the parenthesis is interquartile range and the number before parenthesis is the median value. ${ }^{a}$, Mann-Whitney test. ${ }^{\mathrm{b}}$, Chisquared test. LS, laparoscopic surgery; OS, open surgery; WBC, while blood cells; CCR, creatinine clearance rate.

Table 4 Comparison of intraoperative and postoperative variables between laparoscopic and open surgery group

\begin{tabular}{lccc}
\hline Variable & LS group (N=33) & OS group (N=36) & P value \\
\hline Length of operation (min) & $211.3 \pm 59.1$ & $213.1 \pm 80.6$ & $19 / 17$ \\
Intraoperative hypotension (Y/N) & $7 / 26$ & $18 / 18$ & 0.942 \\
Intraoperative vasopressor intervention (Y/N) & $12 / 21$ & $25 / 11$ & 0.254 \\
Postoperative blood transfusion (Y/N) & $9 / 24$ & $8 / 28$ & $0.0005^{*}$ \\
Postoperative fever (Y/N) & $8 / 25$ & $7 / 29$ & 0.843 \\
Postoperative SIRS (Y/N) & $3 / 30$ & 2 & 0.222 \\
Transfer to ICU (n) & 1 & $7.9 \pm 3.1$ & 0.607 \\
Retroperitoneal drainage (days) & $5.3 \pm 2.6$ & $10.6 \pm 3.5$ \\
Postoperative hospital stay (days) & $7.3 \pm 2.7$ & $0.014^{*}$ \\
\hline
\end{tabular}

*, P<0.05. LS, laparoscopic surgery; OS, open surgery; Y, yes; N, no.

laparoscopic nephrectomy had the advantage of early control of hilar structure and reduction of postoperative pain $(3,7,22)$. The colon was also less likely to be damaged than the transabdominal approach (9). Nevertheless, in the treatment of the right kidney, attention should be paid to prevent the injury of the vena cava (23). And some researchers believed that perirenal abscesses and fixed kidneys were contraindications for surgery, so the selection of surgical methods should be weighed (6).

The significant increase in blood transfusion rate in this study reflected that the difficulty of performing surgery on patients with pyonephrosis accompanied by stone and severe hydronephrosis was significantly higher than that of other benign renal lesions (24). Except for complications requiring blood transfusion, postoperative complications of fever, SIRS and Clavien level 3 or above did not differ between the two groups (20). We thought that severe perirenal infection was exposed during the operation, and local inflammatory factors and bacteriocins entered the blood, resulting in relatively high postoperative infection. Compared with the OS group, the duration of postoperative retroperitoneal drainage and hospital stay in the LS group were significantly shortened. The reduction in length of hospital stay was mainly due to a reduction in the duration of retroperitoneal drainage. The two groups of patients also showed differences in the use of analgesics after the operation; the OS group used more opioid analgesics, while the LS group received more NSAIDs and antispasmodics. Therefore, for complex cases such as non-tuberculous OPNK, retroperitoneal laparoscopic surgery still had 


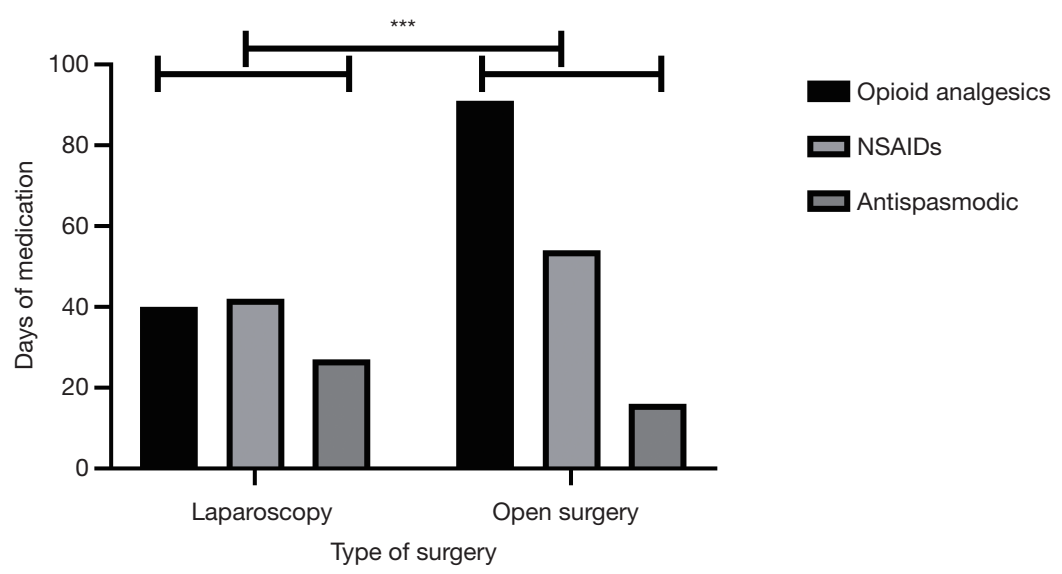

Figure 3 The use of postoperative analgesics in different groups of patients. NSAIDs, non-steroidal anti-inflammatory drugs. ${ }^{* * *}, \mathrm{P}<0.001$.

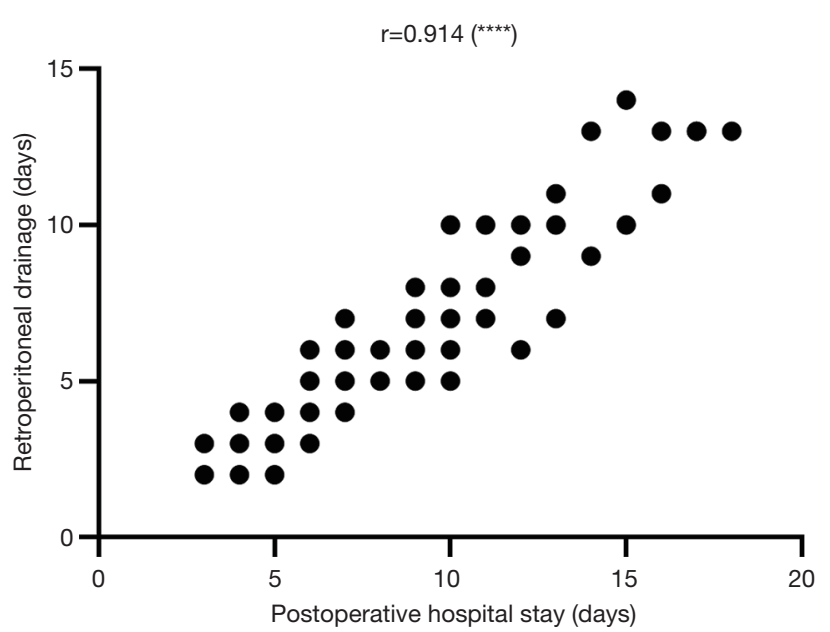

Figure 4 The relationship between the duration of retroperitoneal drainage and postoperative hospital stay. ${ }^{* * *}, \mathrm{P}<0.0001$.

advantages in terms of postoperative efficacy.

Our current research had certain limitations, such as the relatively small number of cases and the inevitable flaws of retrospective research. However, considering the relative rarity of non-tuberculous OPNK and the standardization of single-center surgical procedures, the current research helped to a certain extent in the selection of different surgical procedures and perioperative risk assessment.

\section{Conclusions}

In conclusion, compared with open surgery, laparoscopic surgery had significant advantages in reducing postoperative blood transfusion and the need for opioid analgesics, shortening postoperative surgical drainage and hospital stay for pyonephrotic nephrectomy. When the stone load of pyonephrosis side was less than $280 \mathrm{~mm}^{2}$ and the preoperative $\mathrm{WBC}$ and neutrophil count were within the normal range, laparoscopic operation can be preferred. Open surgery should only be the first choice for pyonephrosis with severe infection and large stone burden.

\section{Acknowledgments}

Funding: This project was supported by the National Natural Science Foundation of China (81900645).

\section{Footnote}

Reporting Checklist: The authors have completed the STROBE reporting checklist. Available at http://dx.doi. org/10.21037/tau-20-1449

Data Sharing Statement: Available at http://dx.doi. org/10.21037/tau-20-1449

Peer Review File: Available at http://dx.doi.org/10.21037/ tau-20-1449

Conflicts of Interest: All authors have completed the ICMJE uniform disclosure form (available at http://dx.doi. org/10.21037/tau-20-1449). The authors have no conflicts of interest to declare.

Ethical Statement: The authors are accountable for all aspects of the work in ensuring that questions related 
to the accuracy or integrity of any part of the work are appropriately investigated and resolved. The study was conducted in accordance with the Declaration of Helsinki (as revised in 2013). It was approved by institutional ethnics committee of Tongji Hospital, Tongji Medical College, Huazhong University of Science and Technology (No. TJA20181101) and individual consent for this retrospective analysis was waived.

Open Access Statement: This is an Open Access article distributed in accordance with the Creative Commons Attribution-NonCommercial-NoDerivs 4.0 International License (CC BY-NC-ND 4.0), which permits the noncommercial replication and distribution of the article with the strict proviso that no changes or edits are made and the original work is properly cited (including links to both the formal publication through the relevant DOI and the license). See: https://creativecommons.org/licenses/by-nc-nd/4.0/.

\section{References}

1. Katz R, Pode D, Golijanin D, et al. Laparoscopic nephrectomy for infected, obstructed and nonfunctioning kidneys. Surg Laparosc Endosc Percutan Tech 2004;14:340-3.

2. Chen Y, Zheng H, Liang G, et al. Comparison of Transperitoneal and Retroperitoneal Laparoscopic Nephrectomy for Nonfunctional Tuberculous Kidneys: A Single-Center Experience. J Laparoendosc Adv Surg Tech A 2018;28:325-9.

3. Garg M, Singh V, Sinha RJ, et al. Prospective randomized comparison of transperitoneal vs retroperitoneal laparoscopic simple nephrectomy. Urology 2014;84:335-9.

4. Challacombe B, Sahai A, Murphy D, et al. Laparoscopic retroperitoneal nephrectomy for giant hydronephrosis: when simple nephrectomy isn't simple. J Endourol 2007;21:437-40.

5. Angerri O, Lopez JM, Sanchez-Martin F, et al. Simple Laparoscopic Nephrectomy in Stone Disease: Not Always Simple. J Endourol 2016;30:1095-8.

6. Manohar T, Desai M, Desai M. Laparoscopic nephrectomy for benign and inflammatory conditions. J Endourol 2007;21:1323-8.

7. Hemal AK, Mishra S. Retroperitoneoscopic nephrectomy for pyonephrotic nonfunctioning kidney. Urology 2010;75:585-8.

8. Tepeler A, Akman T, Tok A, et al. Retroperitoneoscopic nephrectomy for non-functioning kidneys related to renal stone disease. Urol Res 2012;40:559-65.

9. Kaba M, Pirincci N, Taken K, et al. Laparoscopic transperitoneal nephrectomy in non-functioning inflammatory kidneys with or without renal stone. Eur Rev Med Pharmacol Sci 2015;19:4457-61.

10. Yucel C, Ulker V, Kisa E, et al. Laparoscopic Transperitoneal Nephrectomy in Non-functioning Severe Hydronephrotic Kidneys With or Without Renal Stone. Cureus 2018;10:e3729.

11. Zhang S, Luo $\mathrm{Y}$, Wang C, et al. Open surgery versus retroperitoneal laparoscopic nephrectomy for renal tuberculosis: a retrospective study of 120 patients. PeerJ 2016;4:e2708.

12. Kim HH, Lee KS, Park K, et al. Laparoscopic nephrectomy for nonfunctioning tuberculous kidney. J Endourol 2000;14:433-7.

13. Hemal AK, Gupta NP, Kumar R. Comparison of retroperitoneoscopic nephrectomy with open surgery for tuberculous nonfunctioning kidneys. J Urol 2000;164:32-5.

14. Gill IS, Schweizer D, Hobart MG, et al. Retroperitoneal laparoscopic radical nephrectomy: the Cleveland clinic experience. J Urol 2000;163:1665-70.

15. Pearse HD, Barry JM, Houghton DC, et al. Nonfunctioning kidney. J Urol 1979;121:228-31.

16. Mao $\mathrm{S}$, Jiang $\mathrm{H}, \mathrm{Wu} Z$, et al. Urolithiasis: the most risk for nephrectomy in nonrenal tumor patients. J Endourol 2012;26:1356-60.

17. Yasui T, Iguchi M, Suzuki S, et al. Prevalence and epidemiological characteristics of urolithiasis in Japan: national trends between 1965 and 2005. Urology 2008;71:209-13.

18. Kurt O, Buldu I, Turan C, et al. Does laparoscopic transperitoneal simple nephrectomy for inflammatory and non-inflammatory kidneys differ? Springerplus 2016;5:1358.

19. Guillonneau B, Abbou CC, Doublet JD, et al. Proposal for a "European Scoring System for Laparoscopic Operations in Urology". Eur Urol 2001;40:2-6; discussion 7.

20. Naghiyev R, Imamverdiyev S, Efendiyev E, et al. Laparoscopic transperitoneal and retroperitoneal simple nephrectomy: The impact of etiological factors of the results of surgical treatment. Turk J Urol 2017;43:319-24.

21. Kumar M, Kumar R, Hemal AK, et al. Complications of retroperitoneoscopic surgery at one centre. Bju Int 2001;87:607-12.

22. Berglund RK, Gill IS, Babineau D, et al. A prospective comparison of transperitoneal and retroperitoneal laparoscopic nephrectomy in the extremely obese patient. 
Bju Int 2007;99:871-4.

23. Lima M, Miyaoka R, Moro J, et al. Laparoscopic nephrectomy for xanthogranulomatous pyelonephritis-are there predictive factors for success? Clinics (Sao Paulo) 2012;67:907-9.

Cite this article as: Wang X, Tang K, Chen Z, Liu H, Peng E, Xia D. Retroperitoneoscopic nephrectomy versus open surgery for non-tuberculous pyonephrotic nonfunctioning kidney: a single-center experience. Transl Androl Urol 2021;10(3):12121221. doi: $10.21037 / \operatorname{tau}-20-1449$
24. Zelhof B, McIntyre IG, Fowler SM, et al. Nephrectomy for benign disease in the UK: results from the British Association of Urological Surgeons nephrectomy database. BJU Int 2016;117:138-44. 